Received: 7 January 2018

Accepted: 22 June 2018

Published online: 10 July 2018

\section{OPEN A profiling analysis of contributions of cigarette smoking, dietary calcium intakes, and physical activity to fragility fracture in the elderly}

Thuy T. Pham ${ }^{1,2}$, Diep N. Nguyen ${ }^{1}$, Eryk Dutkiewicz ${ }^{1}$, Jacqueline R. Center $\mathbb{D}^{2,3}$, John A. Eisman ${ }^{2,3,5}$ \& Tuan V. Nguyen ${ }^{1,2,3,4,5}$

Fragility fracture and bone mineral density (BMD) are influenced by common and modifiable lifestyle factors. In this study, we sought to define the contribution of lifestyle factors to fracture risk by using a profiling approach. The study involved 1683 women and 1010 men (50+ years old, followed up for up to 20 years). The incidence of new fractures was ascertained by X-ray reports. A "lifestyle risk score" (LRS) was derived as the weighted sum of effects of dietary calcium intake, physical activity index, and cigarette smoking. Each individual had a unique LRS, with higher scores being associated with a healthier lifestyle. Baseline values of lifestyle factors were assessed. In either men or women, individuals with a fracture had a significantly lower age-adjusted LRS than those without a fracture. In men, each unit lower in LRS was associated with a $66 \%$ increase in the risk of total fracture (nonadjusted hazard ratio [HR] 1.66; $95 \% \mathrm{Cl}, 1.26$ to 2.20) and still significant after adjusting for age, weight or BMD. However, in women, the association was uncertain (HR 1.30; 95\% Cl, 1.11 to 1.53). These data suggest that unhealthy lifestyle habits are associated with an increased risk of fracture in men, but not in women, and that the association is mediated by BMD.

Osteoporosis is a common metabolic bone disorder characterized by reduced bone strength and increased fracture risk. The disease affects more than $30 \%$ women and $15 \%$ men aged 50 years and older ${ }^{1}$. Bone strength is primarily reflected by bone mineral density (BMD), which is measured by the dual-energy X-ray absorptiometry (DXA) technology. The variation in BMD can account for up to $60 \%$ of the variance in bone strength ${ }^{2}$. Therefore, $\mathrm{BMD}$ is one of the most important predictors of fracture; each standard deviation (SD) reduction in BMD is associated with 2 -fold increase in the risk of fracture ${ }^{3}$. Thus, osteoporosis has been operationally defined in terms of $\mathrm{BMD}^{2,3}$.

The variation in BMD is determined by multiple factors, including genetic and environmental factors ${ }^{4,5}$. Among environmental factors, lifestyle factors exert considerable effects on $\mathrm{BMD}^{6-12}$. Smoking is a key environmental risk factor because it is significantly associated with decreased hip BMD in old age ${ }^{6}$, but the effect of cigarette smoking on fracture is reversible ${ }^{8}$, A physically active lifestyle and stable weight are associated with reduced bone loss, and thus contribute to the reduction of fracture risk ${ }^{11}$. In the elderly, physical activity was associated with a $20-40 \%$ reduced risk of hip fracture (relative to sedentary subjects) ${ }^{12}$. Several studies have found that adequate dietary calcium intakes were associated with better bone health (i.e., higher bone mass and reduced fracture risk) ${ }^{13,14}$. Nevertheless, a recent meta-analysis has suggested that the effect of calcium intakes was uncertain ${ }^{15}$. Alcohol consumption has also been suggested as a relevant factor for bone health; however, the impact of alcohol consumption on bone health is complex and may be dependent on other factors ${ }^{16}$.

${ }^{1}$ Engineering and Information Technology, University of Technology, Sydney, 2006, Australia. ${ }^{2}$ Bone Biology Division, Garvan Institute of Medical Research, Sydney, 2006, Australia. ${ }^{3}$ St Vincent Clinical School, UNSW, Sydney, Australia. ${ }^{4}$ School of Public Health and Community Medicine, UNSW, Sydney, Australia. ${ }^{5}$ Notre Dame University School of Medicine, Sydney, Australia. Correspondence and requests for materials should be addressed to T.V.N. (email: t.nguyen@garvan.org.au) 


\begin{tabular}{|c|c|c|c|c|c|}
\hline & \multirow[b]{2}{*}{ Predictor } & \multirow[b]{2}{*}{ Unit } & \multirow{2}{*}{\begin{tabular}{|l|} 
No fracture \\
$\left(\right.$ mean, $\left.\mathrm{SD}^{\mathrm{b}}\right)$ \\
\end{tabular}} & \multirow{2}{*}{\begin{tabular}{|l|} 
Fracture \\
(mean, SD) \\
\end{tabular}} & \multirow[b]{2}{*}{$\mathbf{P}^{\mathbf{a}}$} \\
\hline & & & & & \\
\hline \multirow{12}{*}{ Men } & $\mathrm{N}$ & people & 963 & 206 & \\
\hline & Age & years & $68.38(6.18)$ & $71.73(6.73)$ & $* * *$ \\
\hline & Weight & $\mathrm{kg}$ & $81.41(13.74)$ & $77.17(12.21)$ & $* * *$ \\
\hline & Height & $\mathrm{cm}$ & $173.79(6.82)$ & $172.26(6.32)$ & $* *$ \\
\hline & Body mass index & $\mathrm{kg} / \mathrm{m}^{2}$ & $26.9(3.99)$ & $25.94(3.79)$ & $* *$ \\
\hline & Smoking & Packyears & $21.14(29.32)$ & $30.06(36.44)$ & $* * *$ \\
\hline & Smokers & Yes/No (n; \%) & $593(61.58 \%)$ & $138(66.99 \%)$ & \\
\hline & Max alcohol intakes & drinks/week ${ }^{c}$ & $1.33(2.34)$ & $1.54(3.70)$ & \\
\hline & Dietary calcium intakes & g/day & $699.12(358.85)$ & $621.01(377.11)$ & $* *$ \\
\hline & Physical activity index & hours/week & $33.27(5.43)$ & $32.47(5.60)$ & \\
\hline & Lumbar spine $B M D^{d}$ & $\mathrm{~g} / \mathrm{cm}^{2}$ & $0.94(0.14)$ & $0.86(0.16)$ & $* * *$ \\
\hline & Femoral neck BMD & $\mathrm{g} / \mathrm{cm}^{2}$ & $1.28(0.21)$ & $1.17(0.20)$ & $* * *$ \\
\hline \multirow{12}{*}{ Women } & $\mathrm{N}$ & people & 1362 & 597 & \\
\hline & Age & years & $67.78(6.95)$ & $70.96(7.27)$ & $* * *$ \\
\hline & Weight & $\mathrm{kg}$ & $68.51(13.39)$ & $64.88(13.03)$ & $* * *$ \\
\hline & Height & $\mathrm{cm}$ & $160.47(6.09)$ & $159.08(6.69)$ & $* * *$ \\
\hline & Body mass index & $\mathrm{kg} / \mathrm{m}^{2}$ & $26.61(5.04)$ & $25.59(4.9)$ & $* * *$ \\
\hline & \begin{tabular}{|l|} 
Smoking \\
\end{tabular} & Packyears & $6.54(14.81)$ & $7.98(16.31)$ & $*$ \\
\hline & Smokers & Yes/No (n; \%) & $406.00(29.81 \%)$ & $194.00(32.50 \%)$ & $* * *$ \\
\hline & Max alcohol intake & drinks/week $^{c}$ & $0.41(0.78)$ & $0.35(0.8)$ & \\
\hline & Dietary calcium intakes & g/day & 718.85 (394.59) & $689.58(376.85)$ & \\
\hline & Physical activity index & hours/week & $30.75(2.89)$ & $30.41(3)$ & $*$ \\
\hline & Lumbar spine BMD & $\mathrm{g} / \mathrm{cm}^{2}$ & $0.83(0.14)$ & $0.75(0.13)$ & $* * *$ \\
\hline & Femoral neck BMD & $\mathrm{g} / \mathrm{cm}^{2}$ & $1.09(0.20)$ & $0.98(0.19)$ & $* * *$ \\
\hline
\end{tabular}

Table 1. Characteristics of individuals (baseline). 'Significane codes: ‘***' is $<0.001$; ‘**' 0.01 ; ‘*’ 0.05 ; '. 0.1 ; “” $>0: 1$. ${ }^{b}$ SD: standard deviation. ${ }^{c}$ Alcohol consumption intakes were assessed by standard drinks per week. 1 standard drink $=$ a glass of wine or a middie of beer or a nip of spirits. ${ }^{\mathrm{d} B M D}$ : Bone mineral density.

Earlier studies generally examined the effect of lifestyle factors individually. However, these factors tend to co-vary within an individual; smokers tend to have reduced physical health and athletic performance ${ }^{17}$, and individuals who exercise are less likely to smoke, and engaging in exercise may help quit smoking ${ }^{18}$. Similarly, adequate calcium intakes may maximize the positive effect of physical activity on bone health during the growth period of individuals ${ }^{19}$. Therefore, a more comprehensive and holistic approach may offer a better method to evaluate the magnitude of effects of multiple lifestyle factors on BMD or fracture burden.

We hypothesize that a linearly combined effect of low dietary calcium intake, cigarette smoking, and low physical activity is associated with a greater risk of fracture and lower BMD. The present study was designed to test the hypothesis by pursing the following two specific aims: (i) to quantify the contribution of each lifestyle factor to the variation in fracture risk; and (ii) to define the association between combined effects of lifestyle factors on fracture risk.

\section{Results}

Baseline characteristics. The study ultimately included 1960 women and 1170 men whose baseline characteristics are shown in Table 1. The average (SD) age of participants was 69 (6.18) years. The individuals' health status had been followed monitored for between 0.1 and 20 years (average of 12 years). During the follow-up period, 597 women (30.5\%) and 206 men (17.6\%) had reported to sustaine a fragility fracture. Among of 803 fracture cases, 165 occurred at the hip and 331 at vertebrae in either women or men.

As expected, men and women with a fracture were older than those without a fracture. Individuals with fracture also had lower body weight, lower body mass index, lower BMD at the lumbar spine (LSBMD) or femoral neck (FNBMD). In men, fracture cases had significantly lower dietary calcium intakes and higher cigarette intakes (pack-years) than those without a fracture. In women, physical activity index was significantly lower in the fracture group compared with the non-fracture group.

Lifestyle factors and BMD. Figure 1 shows the relationship between individual lifestyle factors and femoral neck BMD. In either men or women, greater dietary calcium intakes, and greater alcohol intakes were each associated with higher femoral neck BMD. Moreover, greater tobacco intakes were associated with lower femoral neck BMD. The magnitudes of association between each of the factors and femoral neck BMD or lumbar spine BMD are shown in terms of regression coefficients (Table 2).

When physical activity, dietary calcium intakes and smoking (packyears) were combined into a "lifestyle risk score" (LRS) by using the regression coefficients, greater LRS was significantly associated with higher FNBMD 
(Fig. 2a) and $L S B M D$ (Fig. 2b) in men and women. In the linear regression analysis (Table 2) greater dietary calcium intakes, greater physical activity index, and higher alcohol intakes were each significantly associated with greater femoral neck BMD in men and women. We found that each unit increase in LRS was associated with $0.085 \mathrm{~g} / \mathrm{cm}^{2}(P<0.0001)$ greater femoral neck BMD in men or $0.088 \mathrm{~g} / \mathrm{cm}^{2}(P<0.0001)$ in women. The association between LRS and lumbar spine BMD remained statistically significant after adjusting for age and weight (Table 2). Moreover, greater tobacco intakes were associated with lower femoral neck BMD.

Lifestyle factors and fracture. For the ease of interpretation, we classified LRS into "low" $(L R S<0)$ or "high" ( $L R S \geq 0)$ based on the median value. Individuals with low LRS had a greater risk of fracture than those with high LRS, and the difference was more pronounced in men (Fig. 3a) than in women (Fig. 3b). The association between LRS and fracture risk was also observed for hip fracture in men (Fig. 3c) and in women (Fig. 3d), for vertebral fracture in men (Fig. 3e) and in women (Fig. 3f).

Table 3 shows that low-LRS men had a $66 \%$ increase in the hazard of total fracture (HR: 1.66; 95\% confidence interval (CI), 1.26 to 2.20 ). This association in men was more evident in for vertebral fracture (HR: $2.52 ; 95 \% \mathrm{CI}$, 1.57 to $4.05, P<0.001$ ), but not for hip fracture (HR: $1.70 ; 95 \% \mathrm{CI}, 0.90$ to $3.19, P=0.1$ ). In women, lower LRS was associated with a $30 \%$ increase in the hazard of total fracture (HR: $1.30 ; 95 \% \mathrm{CI}, 1.11$ to $1.53, P=0.002$ ), but the association was more pronounced for hip fracture (HR: $1.91 ; 95 \% \mathrm{CI}, 1.31$ to $2.79, P=0.001$ ). After adjusting for age and weight or BMI (Table 4), the HR of fracture at any site was 1.61 (95\% CI, 1.22 to $2.13, P=0.001)$ for men and $1.13(95 \% \mathrm{CI}, 0.96$ to $1.33, P=0.155)$ for women.

\section{Discussion}

Osteoporosis can be considered a lifestyle disease because its susceptibility is linked to unhealthy lifestyle. Cigarette smoking, lack of physical activity, and low dietary calcium intakes are each associated with increased risks of osteoporosis and fracture. However, the association between individual lifestyle factors and fracture risk is not always consistent, due largely to sample size issue, and more importantly, to the covariation between lifestyle factors. In this study, we recognized the covariation by creating a profile for each individual, and we found that individuals with an "unhealthy" lifestyle profile had lower BMD and higher risk of fracture than those with a better healthy lifestyle. This finding deserves further elaboration.

It has long been known that lifestyle factors contribute to the development of osteoporosis and increased risk of fracture. Among lifestyle factors that are associated with osteoporosis, cigarette smoking is a prominent risk factor. Cigarette smoking was first linked to osteoporosis more than 40 years ago ${ }^{20}$. In a meta-analysis of 29 studies, postmenopausal bone loss was greater in current smokers than non-smokers, with 6\% difference in BMD being observed by the age of 80 years old ${ }^{21}$. In women, the cumulative risk of hip fracture is $7 \%$ and $15 \%$ higher in current smokers than non-smokers aged 85 and 90 years, respectively ${ }^{21}$. In a recent meta-analysis, compared with non-smokers, the risk of osteoporotic fracture was $32 \%$ higher in smoking men and women ${ }^{22}$. In another study, the risk of hip fracture was 1.6-fold higher in men with history of smoking than those without, and cessation of smoking reduced the risk of hip fracture by $27 \%{ }^{23}$. Our present result of the association between cigarette smoking and fracture or BMD further confirms the association reported in the literature.

The association between dietary calcium intakes and bone health is controversial. In this study, we also found that higher dietary calcium intakes were associated with greater BMD and reduced fracture risk, and this association was independent of age and body weight. Our finding is not necessarily consistent with previous studies. Indeed, the association between dietary calcium intakes and BMD or fracture is not always evident. In the Study of Osteoporotic Fractures, milk drinking and dietary calcium intake as estimated by the food-frequency questionnaires, were not significantly associated with fracture ${ }^{24}$. Actually, when compared with the never users, current users of calcium supplements were at greater risk of hip fractures ${ }^{24}$. A population-based study found that the association between dietary calcium intake and fracture risk was not linear ${ }^{25}$. A recent meta-analysis of seven randomised trials found no significant effect of calcium intake on hip fracture risk ${ }^{26}$. Taken together, although calcium is an important factor in bone health, the association between dietary calcium intake and bone density or fracture is less clear in observational studies.

Apart from smoking and dietary calcium intakes, we found that greater physical activity was associated with greater BMD and lower fracture risk. This finding is consistent with the literature ${ }^{27,28}$. A recent meta-analysis with 22 prospective cohort studies found that individuals with higher level of physical activity had a 30-40\% lower risk of fracture compared to those who had little or no physical activity ${ }^{27}$. The beneficial effect of physical activity on bone health was also evident in a clinical trial in which the risk of overall fracture was $50 \%$ lower in the exercise group than the control ${ }^{28}$.

Whether alcohol consumption has a positive or negative influence on bone health is a contentious issue. In this study, we observed that, to estimate BMD, the maximum alcohol consumption tends to have a non-linear relationship with bone mineral density, as the linear coefficient (against either FNBMD or LSBMD) we found is not consistent for both men and women. Generally, lower alcohol intakes seemed to have positive influence on bone mineral density, but higher alcohol intakes tended to be associated with lower bone mineral density, but the relationship was not consistent for men and women. This observation seems be supported in by earlier works which suggested that the association between the maximum alcohol consumption and BMD or fractures is complex and even may be dependent on other factors ${ }^{16}$. Because of this complex relationship, we did not consider alcohol intakes in the lifestyle profiling in this study.

Cigarette smoking, dietary calcium intakes, and physical activity index are tend to be correlated. The co-occurrence of smoking and physical inactivity has been observed in at least 50 studies $^{29,30}$, with smoking being associated with lower levels of physical activity ${ }^{29}$. Furthermore, smoking individuals with low physical activity index tend to be associated with lower dietary calcium intakes. Therefore, it is logical to consider that these factors simultaneously, even though individually some factors may not be associated with bone health. In this study, we 


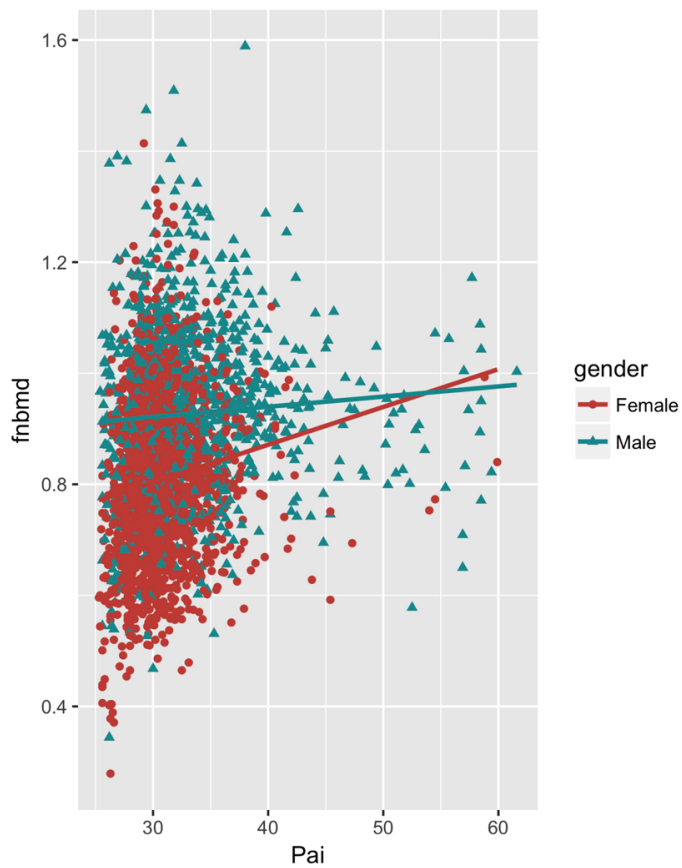

(a) Physical activity index vs FNBMD.

$\mathrm{R}$ squared is 0.0045 (men) or 0.0203 (women).

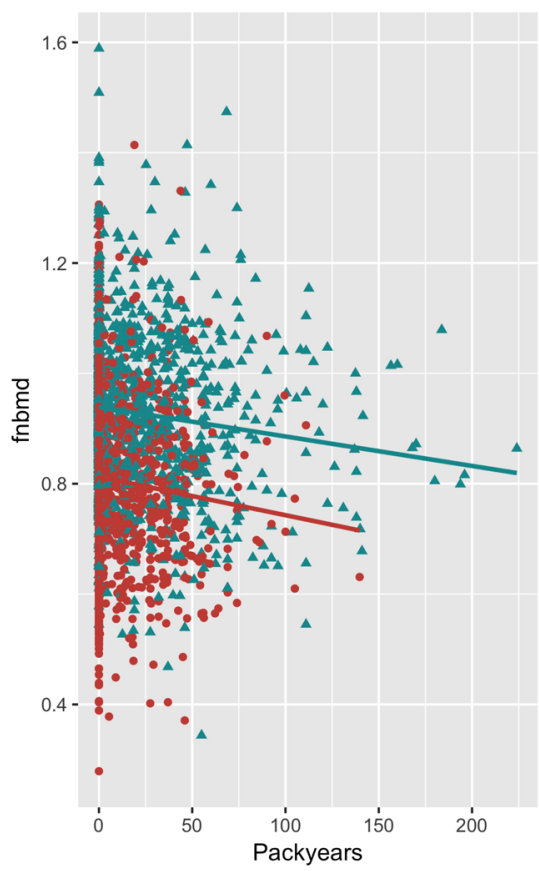

(c) Smoking (packyears) vs FNBMD.

$\mathrm{R}$ squared is 0.0123 (men) or 0.0058 (women).

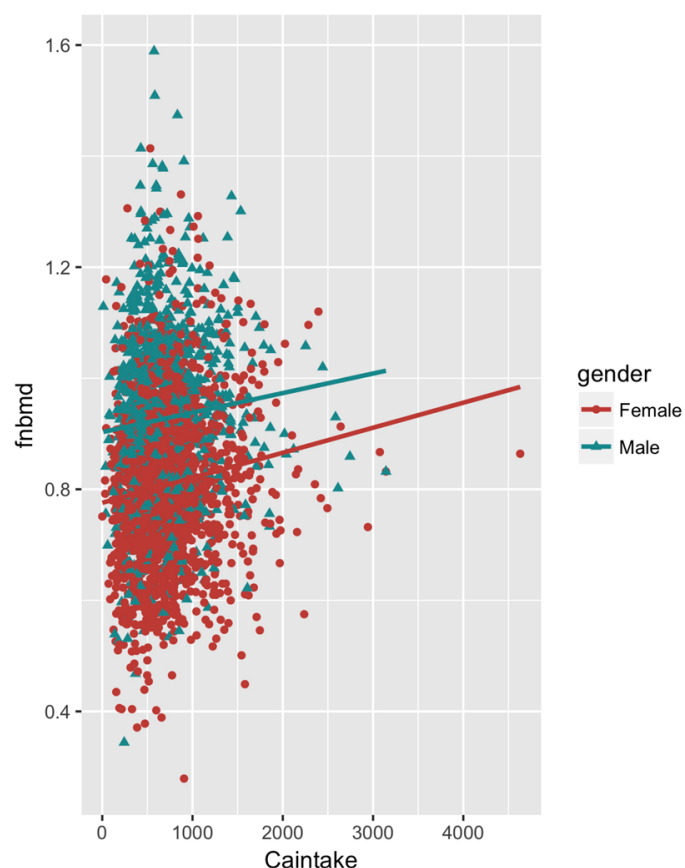

(b) Dietary calcium intake vs FNBMD.

$\mathrm{R}$ squared is 0.0073 (men) or 0.0158 (women).

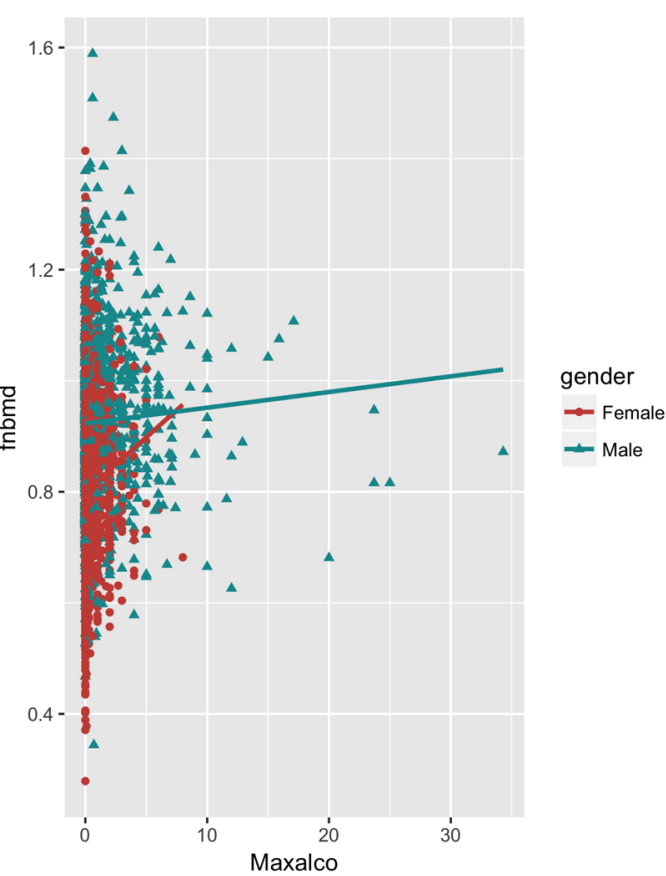

(d) Max alcohol intake vs FNBMD.

$\mathrm{R}$ squared is 0.0025 (men) or 0.0122 (women).

Figure 1. Lifestyle factors against femoral neck bone mineral density (FNBMD). R squared values are reported from corresponding linear regression estimations. The models were note adjusted for age and BMI. (a) Physical activity index vs FNBMD. R squared is 0.0045 (men) or 0.0203 (women). (b) Dietary calcium intake vs FNBMD. R squared is 0.0073 (men) or 0.0158 (women). (c) Smoking (packyears) vs FNBMD. R squared is 0.0123 (men) or 0.0058 (women). (d) Max alcohol intake vs FNBMD. R squared is 0.0025 (men) or 0.0122 (women).

have created a "lifestyle signature" or LRS for each individual based on the magnitude of association between these factors and BMD, and then related LRS with fracture risk. The adverse association between LRS and fracture risk indicates that the cumulative exposure to unhealthy lifestyle had detrimental effect on bone health in both men and women. 


\begin{tabular}{|c|c|c|c|c|}
\hline \multirow[b]{2}{*}{ Predictor } & \multicolumn{2}{|c|}{ Femoral neck (FN) BMD ${ }^{a}$} & \multicolumn{2}{|c|}{ Lumbar spine (LS) BMD } \\
\hline & Coefficient $\pm \mathbf{S E}^{\mathbf{b}}$ & P-value ${ }^{b}$ & Coefficient \pm SE & P-value \\
\hline \multicolumn{5}{|l|}{ Men } \\
\hline Dietary calcium intakes & $0.013 \pm 0.005$ & 0.003 & $0.029 \pm 0.007$ & $<0.001$ \\
\hline Physical activity index & $0.008 \pm 0.003$ & 0.022 & $-0.007 \pm 0.005$ & 0.166 \\
\hline Smoking Packyears & $-0.013 \pm 0.003$ & $<0.001$ & $-0.016 \pm 0.005$ & 0.001 \\
\hline Max alcohol intake & $0.005 \pm 0.003$ & 0.088 & $0.001 \pm 0.004$ & 0.744 \\
\hline LRS $^{c}$ & $0.085 \pm 0.017$ & $<0.001$ & $0.097 \pm 0.017$ & $<0.001$ \\
\hline \multicolumn{5}{|l|}{ Women } \\
\hline Dietary calcium intakes & $0.017 \pm 0.003$ & $<0.001$ & $0.007 \pm 0.004$ & 0.119 \\
\hline Physical activity index & $0.029 \pm 0.005$ & $<0.001$ & $0.013 \pm 0.007$ & 0.044 \\
\hline Smoking Packyears & $-0.016 \pm 0.005$ & 0.001 & $-0.005 \pm 0.007$ & 0.445 \\
\hline Max alcohol intake & $0.035 \pm 0.007$ & $<0.001$ & $0.045 \pm 0.010$ & $<0.001$ \\
\hline LRS $^{c}$ & $0.088 \pm 0.010$ & $<0.001$ & $0.089 \pm 0.035$ & 0.012 \\
\hline
\end{tabular}

Table 2. Lifestyle determinants of BMD using multiple linear regression analysis. ${ }^{a} B M D:$ Bone mineral density. LRS: Lifestyle Risk Score. ${ }^{b}$ SE: standard error. P-value is for the coeffiecients in the linear regression analysis. ${ }^{\mathrm{C}} \mathrm{I}(\mathrm{LRS} / 0.1)$. Notes: Lifestyle predictors are normalized using zscores. Coefficients of linear regression are adjusted by age and body mass index.

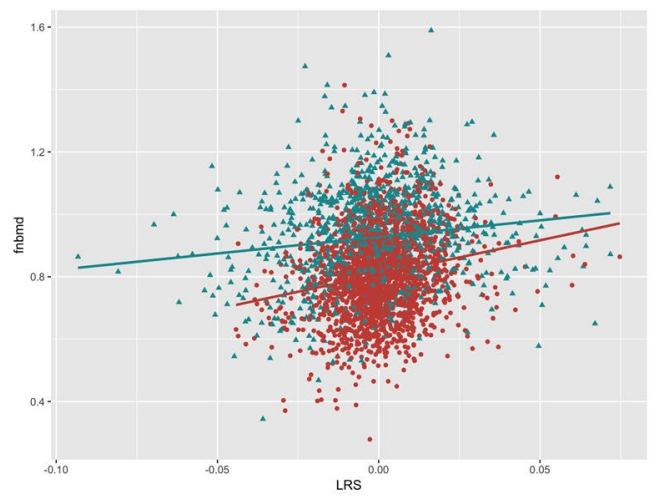

(a) LRS vs FNBMD.

$\mathrm{R}$ squared is 0.0206 (men) or 0.0370 (women).

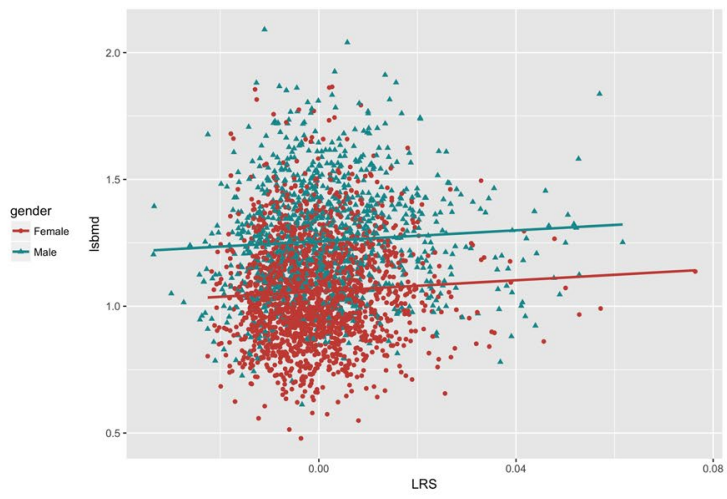

(b) LRS vs LSBMD.

$\mathrm{R}$ squared is 0.0270 (men) or 0.0032 (women).

Figure 2. Lifestyle Risk Score (LRS) against femoral neck BMD (FNBMD) and lumbar spine BMD (LSBMD). $\mathrm{R}$ squared values are reported from corresponding linear regression estimations. The models were note adjusted for age and BMI. (a) LRS vs FNBMD. R squared is 0.0206 (men) or 0.0370 (women). (b) LRS vs LSBMD. R squared is 0.0270 (men) or 0.0032 (women).

Our findings also have important implications for public health and clinical assessment. As lifestyle factors are modifiable, this association implies that improving lifestyle at the individual level can reduce fracture risk. At present, fracture risk assessment is based on clinical risk factors, with modest to good discrimination ${ }^{31}$. This study showed that the incorporation of lifestyle profiling into fracture risk assessment could improve the accuracy of fracture risk prediction, especially for vertebral fractures in men where discrimination based on clinical risk factors is modest.

The present findings must however be considered within the context of strengths and weaknesses of the study. The study was based on a large and well-characterized cohort, with long duration of follow-up that allows better detection of fine associations. We consider that our lifestyle profiling approach is a strength of the study, because with this approach we could simultaneously examined the impact of multiple risk factors on fracture. The ascertainment of fractures by X-ray, not self-reports, represents a strength of our study, because it avoided the problem of misclassification or misdiagnoses. Nevertheless, data concerning lifestyle factors in this study were self-reported, and misclassification and measurement error could be present in the analysis. The dietary calcium data were derived from a food frequency questionnaire with the time-frame of one week which may not reflect the long-term intake. We considered three lifestyle factors in this study, and we therefore could not exclude unmeasured confounding as partial accounting for the findings. Moreover, because the study was an observational investigation, no causal inference could be made on the relationship between lifestyle and fracture.

In conclusion, these data suggest that cumulative exposure to unhealthy lifestyle factors (e.g., smoking, lower physical activity index, and lower dietary calcium intakes) is associated with increased fracture risk, and the association is mainly driven by bone mineral density. Given that lifestyle risk factors are modifiable and that 


\begin{tabular}{|c|c|c|c|c|c|c|}
\hline \multirow[b]{2}{*}{ Fracture Site } & \multirow[b]{2}{*}{ Predictor $^{\mathrm{a}}$} & \multirow[b]{2}{*}{ Unit } & \multicolumn{2}{|l|}{ Men } & \multicolumn{2}{|l|}{ Women } \\
\hline & & & $\mathrm{HR}^{\mathrm{b}}(\mathrm{CI})$ & P-value ${ }^{c}$ & HR (CI) & P-value \\
\hline \multirow{3}{*}{ Any fracture } & LRS & Low/High & $1.66(1.26-2.20)$ & $<0.001$ & $1.30(1.11-1.53)$ & 0.001 \\
\hline & Age & $(+5$ years $)$ & $1.52(1.38-1.69)$ & $<0.001$ & $1.35(1.28-1.43)$ & $<0.001$ \\
\hline & FNBMD $^{\mathrm{d}}$ & $\left(-0.12 \mathrm{~g} / \mathrm{cm}^{2}\right)$ & $0.64(0.56-0.72)$ & $<0.001$ & $0.60(0.55-0.64)$ & $<0.001$ \\
\hline \multirow{3}{*}{ Hip fracture } & LRS & Low/High & $1.70(0.90-3.19)$ & 0.101 & $2.10(1.43-3.07)$ & $<0.001$ \\
\hline & Age & $(+5$ years $)$ & $1.92(1.53-2.40)$ & $<0.001$ & $1.93(1.73-2.16)$ & $<0.001$ \\
\hline & FNBMD & $\left(-0.12 \mathrm{~g} / \mathrm{cm}^{2}\right)$ & $0.31(0.23-0.42)$ & $<0.001$ & $0.30(0.25-0.35)$ & $<0.001$ \\
\hline \multirow{3}{*}{ Vertebral fracture } & LRS & Low/High & $2.52(1.57-4.05)$ & $<0.001$ & $1.16(0.90-1.49)$ & 0.253 \\
\hline & Age & $(+5$ years $)$ & $1.58(1.35-1.86)$ & $<0.001$ & $1.48(1.36-1.61)$ & $<0.001$ \\
\hline & FNBMD & $\left(-0.12 \mathrm{~g} / \mathrm{cm}^{2}\right)$ & $0.48(0.40-0.58)$ & $<0.001$ & $0.50(0.44-0.57)$ & $<0.001$ \\
\hline
\end{tabular}

Table 3. Prediction of fracture risk using univariate Cox Proportional Hazards Analysis. ${ }^{a} L R S$ : Lifestyle Risk Score. LRS in Low/High. Age in ( +5 years). Weight in $(\mathrm{kg})$. BMI in $\left(\mathrm{kg}=\mathrm{m}^{2}\right)$. FNBMD: Femoral neck bone mineral density. ${ }^{\mathrm{b}} \mathrm{HR}$ : Hazard ratio. $95 \% \mathrm{CI}$ : Confidence intervals of the hazard ratio. ${ }^{\mathrm{c} P}$-value: Global statistical significance of the model. 'FNBMD: Femoral neck bone mineral density.

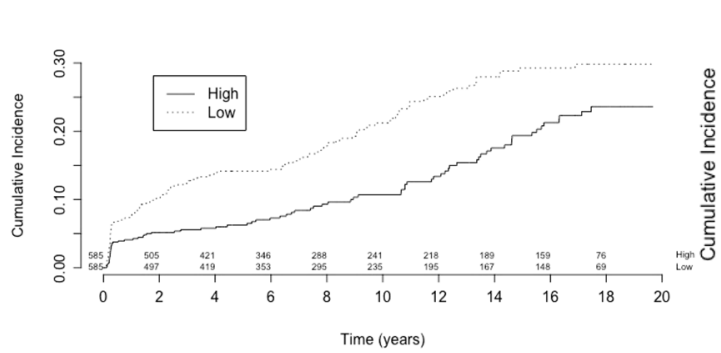

(a) Any fracture in men. $\mathrm{P}=0.0003$.

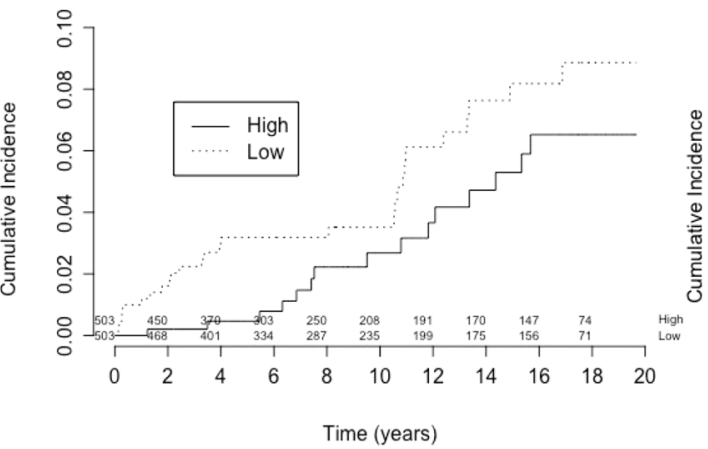

(c) Hip fracture in men. $\mathrm{P}=0.0969$.

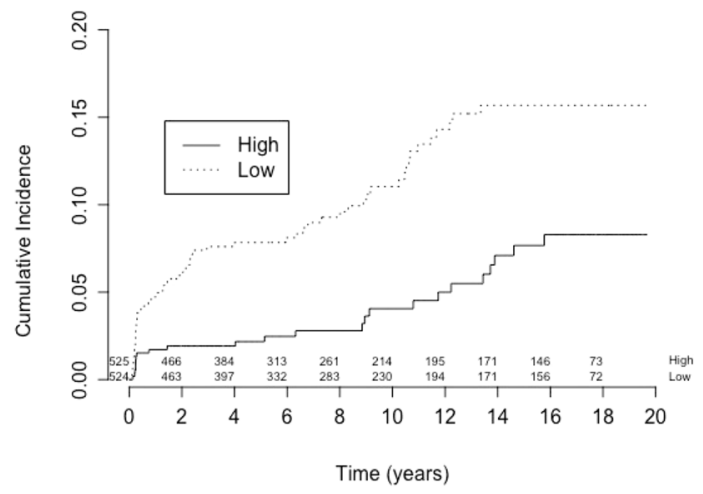

(e) Vertebral fracture in men. $P<0.0001$.

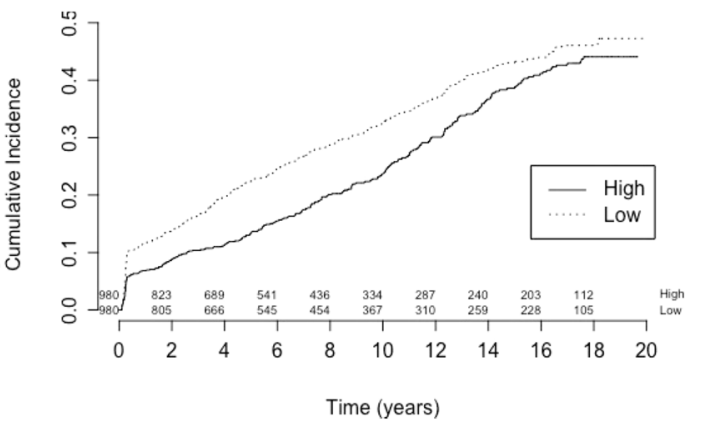

(b) Any fracture in women. $\mathrm{P}=0.0014$.

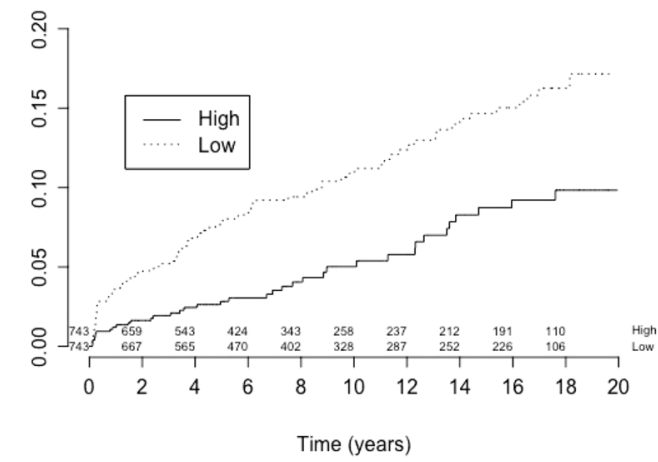

(d) Hip fracture in women. $\mathrm{P}=0.0001$.

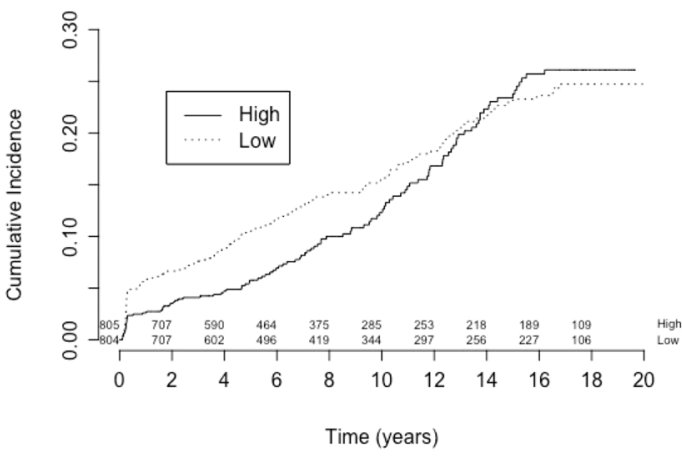

(f) Vertebral fracture in women. $\mathrm{P}=0.253$.

Figure 3. Cumulative Incidence over time (years) in men and women. P: P-value reported in each subplot is for the difference between survival curve by Chisquare test. (a) Any fracture in men. $P=0.0003$. (b) Any fracture in women. $\mathrm{P}=0.0014$. (c) Hip fracture in men. $\mathrm{P}=0.0969$. (d) Hip fracture in women. $\mathrm{P}=0.0001$. (e) Vertebral fracture in men. $P<0.0001$. (f) Vertebral fracture in women. $\mathrm{P}=0.253$. 


\begin{tabular}{|c|c|c|c|c|c|}
\hline \multirow[b]{2}{*}{ Fracture Site } & \multirow[b]{2}{*}{ LRS $^{a}$ adjusted by } & \multicolumn{2}{|l|}{ Men } & \multicolumn{2}{|l|}{ Women } \\
\hline & & $\mathrm{HR}^{\mathrm{b}}(\mathrm{CI})$ & P-value ${ }^{c}$ & HR (CI) & P-value \\
\hline \multirow{5}{*}{ Any fracture } & Age & $1.59(1.20-2.11)$ & 0.001 & $1.12(0.95-1.32)$ & 0.187 \\
\hline & Age + BMI & $1.59(1.20-2.11)$ & 0.001 & $1.13(0.96-1.33)$ & 0.148 \\
\hline & Age + FNBMD & $1.49(1.13-1.98)$ & 0.005 & $1.08(0.91-1.27)$ & 0.387 \\
\hline & Age + Weight & $1.60(1.21-2.12)$ & 0.001 & $1.14(0.96-1.34)$ & 0.131 \\
\hline & Age + Priorfx & $1.60(1.21-2.12)$ & 0.001 & $1.11(0.94-1.31)$ & 0.208 \\
\hline \multirow{5}{*}{ Hip fracture } & Age & $1.59(0.84-2.99)$ & 0.151 & $1.33(0.89-1.97)$ & 0.161 \\
\hline & Age + BMI & $1.62(0.86-3.04)$ & 0.136 & $1.44(0.97-2.15)$ & 0.070 \\
\hline & Age + FNBMD & $1.20(0.63-2.29)$ & 0.573 & $1.19(0.80-1.77)$ & 0.386 \\
\hline & Age + Weight & $1.63(0.87-3.07)$ & 0.129 & $1.43(0.96-2.13)$ & 0.075 \\
\hline & Age + Priorfx & $1.62(0.86-3.05)$ & 0.137 & $1.31(0.88-1.96)$ & 0.178 \\
\hline \multirow{5}{*}{ Vertebral fracture } & Age & $2.36(1.47-3.78)$ & $<0.001$ & $0.94(0.73-1.22)$ & 0.652 \\
\hline & Age + BMI & $2.36(1.47-3.78)$ & $<0.001$ & $0.94(0.73-1.22)$ & 0.750 \\
\hline & Age + FNBMD & $2.37(1.47-3.80)$ & 0.004 & $0.96(0.74-1.24)$ & 0.493 \\
\hline & Age + Weight & $2.36(1.47-3.78)$ & $<0.001$ & $0.94(0.73-1.22)$ & 0.821 \\
\hline & Age + Priorfx & $2.35(1.47-3.78)$ & $<0.001$ & $0.97(0.75-1.26)$ & 0.636 \\
\hline
\end{tabular}

Table 4. Hazard ratio of fracture associated with lifestyle profiling after adjusting for age, body mass index (BMI), femoral neck BMD, and prior fracture. ${ }^{a}$ LRS: Lifestyle Risk Score. LRS in Low/High. Age in (+5 years). Weight in $(\mathrm{kg})$. BMI in $\left(k g=m^{2}\right)$. FNBMD: Femoral neck bone mineral density. ${ }^{b} \mathrm{HR}$ : Hazard ratio. $95 \%$ CI: Confidence intervals of the hazard ratio. ${ }^{\mathrm{C}} \mathrm{P}$-value: Global statistical significance of the model.

fracture prevalence is high in the general population, the present finding implies that the burden of fracture can be reduced at the individual level.

\section{Methods}

Study Design and Setting. This work is part of the Dubbo Osteoporosis Epidemiology Study (DOES), in which the protocol and study design have been described elsewhere ${ }^{32,33}$. Briefly, the Study involved 1581 men and 2095 women who were over 50 years old in Dubbo city and the surrounding districts (NSW, Australia) and were invited to participate in the Study in 1989. After missing data were excluded, data from 1683 women and 1010 men were analysed further. Dubbo city is located approximately $400 \mathrm{~km}$ north-west of Sydney. The city was chosen for this longitudinal study because of several reasons. First, it had population structure which resembled the overall Australian population. Second, its health services are largely self-contained allowing for the ascertainment of health outcomes, which made it become ideal for a prospective epidemiology study. More importantly, the city has 3 radiologists making it ideal for collecting almost all fractures within the area.

At the initial of the study in 1989, the population of Dubbo consisted of approximately 32,000 people, of whom approximately 99\% were Caucasian and about 1\% were Australian Aboriginal. All 1581 men and 2095 women aged 60 years or above as at mid-1989 residing in Dubbo and the two surrounding districts (Geurie and Wongarbon) which had the same postcode as Dubbo were invited to participate in the study. This study represented for $11 \%$ of the entire Dubbo population. Potential participants were chosen from the electoral roll (where enrolment is compulsory in Australia) and media campaigns. The rate of participation was $69 \%$ for women and $58 \%$ for men. Although all eligible men and women within the Dubbo area were invited to participate into the study, all study participants were of Caucasian origin. During follow-up, more participants were collected; however, majority of participants were recruited within the first two years of the commencement. The study's procedure and protocol were approved by the St Vincent's Campus Research Ethics Committee (NSW, Australia), and written informed consent was obtained from each participant. All research in this work were performed in accordance with relevant guidelines/regulations.

Fracture Ascertainment. Non-trauma fracture was the main outcome of this study. The incidence of new fractures was ascertained through X-ray reports (provided by the two local radiology centres, as previously ${ }^{34}$ ). The fracture incidence had been continuously recorded since 1990. Fractures were included only if the report of fracture was definite and occurred with low trauma (e.g., fall from standing heights or less) which was confirmed on interview. Fractures were excluded from analysis if they were resulted from major trauma (e.g., motor vehicle accident), underlying diseases (e.g., cancer or bone-related diseases), or were fractures of the digits, skull, or cervical spine.

Bone Mineral Density. Bone mineral density $\left(\mathrm{BMD}-\mathrm{g} / \mathrm{cm}^{2}\right)$ measurement was performed by dual-energy X-ray absorptiometry (DXA) using a DPX densitometer (GELunar Corp., Madison, WI, USA) in the lumbar spine or femoral neck (radiation dose was $<0.1 p L G y$ ). Coefficient of variation for BMD in our normal subjects is $1-5 \%$ for lumbar spine and $1-3 \%$ for femoral neck ${ }^{34}$.

Physical activity. The quantification of physical activity was based on the method used in the Framingham Massachusetts Heart Study ${ }^{35}$. Five levels of activities were considered: basal activity (sleeping or lying down), 
sedentary (sitting or standing), light (casual walking) moderate (gardening or carpentry), and heavy (lifting or heavy gardening). For each activity level, individuals were asked the average number of hours per week they spent performing each level of activity. The number of hours for each level was then translated to the average amount of oxygen consumed using the Metabolic Equivalent Task (MET) method. MET was derived as the product of the number of hours spent on each level of activity and an intensity weighting factor. The intensity factors for the levels were as follows: $1=$ basal, $1.1=$ sedentary, $1.5=$ light, $2.4=$ moderate, and $5=$ heavy.

Dietary calcium intakes. Dietary calcium intakes were assessed from a 4-day food frequency questionnaire $^{36}$. The amount of dietary calcium intake for each individual was estimated as the sum of calcium content for each food item. The food items included daily intake of milk, bread, yogurt, cheese, ice cream, eggs, fish, cereal foods, fruits and vegetables, alcohols, and others (e.g., chocolate and orange juice). Since milk provides the highest amount of calcium in the diet, multiple questions relating to milk consumption were included to refine the estimate of dietary calcium intakes. The calcium content for each food item was derived from manufacturer's information or from Australian or British tables of food consumption ${ }^{36}$.

Lifestyle Factors. Alcohol intakes were assessed by the number of standard drinks ( $g /$ day). For example, a middie standard of beer, a standard glass of wine, or a nip of spirits equals to $10 \mathrm{~g}$. Smoking was recorded as the number of cigarettes smoked per day, then the cumulative exposure was determined as the number of packyears in our analysis. Dietary calcium intakes were estimated as $m g /$ day. Physical activity was quantified by the metabolic equivalent task (MET) which is the sum of products of the time spent on each activity level and a weighting factor derived from estimated oxygen consumption (i.e. sleep $=1.0$, sedentary $=1.1$, light activity $=1.5$, moderate activity $=2.4$, heavy activity $=5.0$ ). The activities considered in the study were the number of hours of sleep per night, the number of hours spending on writing, reading, sitting, shower, grooming, cleaning, laundry, shopping, gardening, mowing lawn, manual labour digging, recreational activities (e.g., fishing, playing musical instrument, dance, or jogging), and occupational activities.

Lifestyle Profiling. We constructed a single score called "lifestyle risk score" (LRS) that represented a lifestyle profiling for an individual. LRS is a linear summation of effects of smoking (packyears), dietary calcium intake, and physical activity index as follows.

$$
L R S=\alpha_{\text {smoking }} \times \text { packyears }+\alpha_{\text {pai }} \times \text { pai }+\alpha_{c a} \times \text { Calcium }
$$

where packyears is smoking consumption, pai is physical activity index, and Calcium is the amount of dietary calcium intakes and their linear regression coefficients used to estimate BMD: $\alpha_{\text {smoking }}, \alpha_{\text {pai }}$, and $\alpha_{c a}$, respectively. LRS was normalized by z-score so that it has zero mean and unit variance.

Data Analysis. We first used the multiple linear regression model to evaluate the association between lifestyle factors and BMD. The model was adjusted for age and body mass index because the two covariates are known to be associated with BMD. We then used the Cox's proportional hazards model ${ }^{37}$ to evaluate the relationship between LRS and fracture risk. In the multivariable model, the hazard of fracture at time $t, H(t)$, was modeled as a function of background risk, $H_{0}(t)$, and effects of LRS and covariates $\mathrm{X}: H(t)=H_{0}(t) \times \exp (\beta X)$ where $\beta$ is the regression coefficient of $X$ to estimate fracture. Since age and femoral neck BMD are associated with fracture risk $^{38,39}$, these two factors are considered covariates in the multi-variable analysis with Cox's model. The magnitude of association between LRS and fracture risk was assessed by the hazard ratio (HR) and its $95 \%$ confidence interval (CI).

Data Availability. The datasets generated during and/or analysed during the current study are available from the corresponding author on reasonable request.

\section{References}

1. Nguyen, N. D., Ahlborg, H. G., Center, J. R., Eisman, J. A. \& Nguyen, T. V. Residual lifetime risk of fractures in women and men. J. Bone Min. Res. 22, 781-788 (2007).

2. World Health Organization. Assessment of fracture risk and its application to screening for postmenopausal osteoporosis. WHO Tech. Rep. Ser. 843 Geneve (1994).

3. Kanis, J. et al. Ten year probabilities of osteoporotic fractures according to BMD and diagnostic thresholds. Osteoporos. Int. 12, 989-995 (2001)

4. Estrada, K. et al. Genome-wide meta-analysis identifies 56 bone mineral density loci and reveals 14 loci associated with risk of fracture. Nat. Genet. 44, 491-501 (2012).

5. Nguyen, T. V. \& Eisman, J. A. Genetic profiling and individualized assessment of fracture risk. Nat. Rev. Endocrinol. 9, 153-161 (2013).

6. Hollenbach, K. A., Barrett-Connor, E., Edelstein, S. L. \& Holbrook, T. Cigarette smoking and bone mineral density in older men and women. Am. J. Public Heal. 83, 1265-1270 (1993).

7. Vestergaard, P. \& Mosekilde, L. Fracture risk associated with smoking: a meta-analysis. J. Intern. Med. 254, 572-583 (2003).

8. Yoon, V., Maalouf, N. \& Sakhaee, K. The effects of smoking on bone metabolism. Osteoporos Int. 23, 2081-2092 (2012).

9. Berg, K. M. et al. Association between alcohol consumption and both osteoporotic fracture and bone density. Am. J. Med 121, 406-418 (2008)

10. Paccou, J. et al. Relationships between bone geometry, volumetric bone mineral density and bone microarchitecture of the distal radius and tibia with alcohol consumption. Bone 78, 122-129 (2015).

11. Nguyen, T., Sambrook, P. \& Eisman, J. Bone loss, physical activity, and weight change in elderly women: the Dubbo Osteoporosis Epidemiology Study. J. Bone Min. Res. 13, 1458-1467 (1998).

12. Gregg, E. W., Pereira, M. A. \& Caspersen, C. J. Physical activity, falls, and fractures among older adults: a review of the epidemiologic evidence. J. Am. Geriatr. Soc. 48, 883-893 (2000) 
13. Sanders, K. M. et al. Calcium and bone health: position statement for the Australian and New Zealand Bone and Mineral Society, Osteoporosis Australia and the Endocrine Society of Australia. Med J Aust 190, 316-20 (2009).

14. Weaver, C. et al. The national osteoporosis foundation's position statement on peak bone mass development and lifestyle factors: a systematic review and implementation recommendations. Osteoporos Int. 27, 1281-1386 (2016).

15. Bolland, M. J. et al. Calcium intake and risk of fracture: systematic review. BMJ. 351, h4580 (2015).

16. Gaddini, G. W., Turner, R. T., Grant, K. A. \& Iwaniec, U. T. Alcohol: a simple nutrient with complex actions on bone in the adult skeleton. Alcohol. Clin. Exp. Res. 40, 657-671 (2016).

17. US Department of Health and Human Services et al. The health consequences of smoking: A report of the surgeon general (2004).

18. Ussher, M. H., Taylor, A. H., West, R. \& McEwen, A. Does exercise aid smoking cessation? a systematic review. Addict. 95, 199-208 (2000).

19. Specker, B. \& Binkley, T. Randomized trial of physical activity and calcium supplementation on bone mineral content in 3-to 5-yearold children. J. Bone Min. Res. 18, 885-892 (2003).

20. Daniell, H. W. Osteoporosis of the slender smoker: vertebral compression fractures and loss of metacarpal cortex in relation to postmenopausal cigarette smoking and lack of obesity. Arch. Intern. Med. 136, 298-304 (1976).

21. Law, M. R. \& Hackshaw, A. K. A meta-analysis of cigarette smoking, bone mineral density and risk of hip fracture: recognition of a major effect. BMJ. 315, 841-846 (1997).

22. Kanis, J. A. et al. Assessment of fracture risk. Osteoporos Int. 16, 581-589 (2005).

23. Høidrup, S. et al. Tobacco smoking and risk of hip fracture in men and women. Int. J. Epidemiol. 29, 253-259 (2000).

24. Cumming, R. G. et al. Calcium intake and fracture risk: results from the study of osteoporotic fractures. Am. J. Epidemiol 145, 926-934 (1997).

25. Warensiö, E. et al. Dietary calcium intake and risk of fracture and osteoporosis: prospective longitudinal cohort study. BMJ. 342, d1473 (2011)

26. Bischoff-Ferrari, H. A. et al. Calcium intake and hip fracture risk in men and women: a meta-analysis of prospective cohort studies and randomized controlled trials. Am. J. Clin. Nutr. 86, 1780-1790 (2007).

27. Qu, X. et al. Association between physical activity and risk of fracture. J. Bone Min. Res. 29, 202-211 (2014).

28. Kemmler, W., Häberle, L. \& Von Stengel, S. Effects of exercise on fracture reduction in older adults. Osteoporos Int. 24, 1937-1950 (2013).

29. Angus, R., Sambrook, P., Pocock, N. \& Eisman, J. Dietary intake and bone mineral density. Bone Min. 4, 265-277 (1988).

30. Kaczynski, A. T., Manske, S. R., Mannell, R. C. \& Grewal, K. Smoking and physical activity: a systematic review. Am. J. Heal. Behav 32, 93-110 (2008).

31. Nguyen, T. V. \& Eisman, J. A. Genetic profiling and individualized assessment of fracture risk. Nat. Rev. 9, 153-161 (2013).

32. Simons, L. A. et al. The Dubbo study: an Australian prospective community study of the health of elderly. Intern. Medicine J. 20, 783-789 (1990).

33. Nguyen, T. et al. Prediction of osteoporotic fractures by postural instability and bone density. BMJ. 307, 1111-1115 (1993).

34. Nguyen, T., Sambrook, P. \& Eisman, J. Sources of variability in bone mineral density measurements: implications for study design and analysis of bone loss. J. Bone Min. Res. 12, 124-135 (1997).

35. Kannel, W. B. \& Sorlie, P. Some health benefits of physical activity: The framingham study. Arch. Intern. Medicine 139, 857-861 (1979).

36. Angus, R., Sambrook, P., Pocock, N. \& Eisman, J. A simple method for assessing calcium intake in caucasian women. J. Am. Diet. Assoc. 89, 209-214 (1989).

37. Cox, D. R. Regression models and life-tables. In Breakthroughs in Statistics, 527-541 (Springer, 1992).

38. Nguyen, N. D., Frost, S., Center, J. R., Eisman, J. A. \& Nguyen, T. Development of a nomogram for individualizing hip fracture risk in men and women. Osteoporos Int. 18, 1109-1117 (2007).

39. Nguyen, N. D., Frost, S. A., Center, J., Eisman, J. A. \& Nguyen, T. V. Development of prognostic nomograms for individualizing 5-year and 10-year fracture risks. Osteoporos Int. 19, 1431-1444 (2008).

\section{Acknowledgements}

We also appreciate the invaluable help of the staff of Dubbo Base Hospital. We thank the IT group of the Garvan Institute of Medical Research for the management of the database. The Dubbo Osteoporosis Epidemiology Study was supported by the Australian National Health and Medical Research Council, Grant Number APP1031494 (CIA T. Nguyen).

\section{Author Contributions}

T.T.P. and T.V.N. had full access to the whole data set and takes responsibility for the integrity of the data and the accuracy of the data analysis. Study conception: T.V.N., J.A.E. and T.T.P. Study design and data collection: T.V.N., J.A.E. and J.R.C. Data analysis: T.T.P., D.N.N., E.D. and T.V.N. Interpretation of results: T.V.N., J.A.E., J.R.C. and T.T.P. Drafting manuscript: T.T.P., D.N.N., E.D. and T.V.N. reviewed the manuscript. Final approval of manuscript content: T.T.P., J.A.E., J.R.C. and T.V.N.

\section{Additional Information}

Competing Interests: The authors declare no competing interests.

Publisher's note: Springer Nature remains neutral with regard to jurisdictional claims in published maps and institutional affiliations.

Open Access This article is licensed under a Creative Commons Attribution 4.0 International License, which permits use, sharing, adaptation, distribution and reproduction in any medium or format, as long as you give appropriate credit to the original author(s) and the source, provide a link to the Creative Commons license, and indicate if changes were made. The images or other third party material in this article are included in the article's Creative Commons license, unless indicated otherwise in a credit line to the material. If material is not included in the article's Creative Commons license and your intended use is not permitted by statutory regulation or exceeds the permitted use, you will need to obtain permission directly from the copyright holder. To view a copy of this license, visit http://creativecommons.org/licenses/by/4.0/.

(c) The Author(s) 2018 